\title{
Developmental toxicity of dimethachlor during zebrafish embryogenesis mediated by apoptosis and oxidative stress
}

\author{
Garam An ${ }^{1}$, Hahyun Park ${ }^{1}$, Gwonhwa Song ${ }^{1, *}$ and Whasun Lim²,* \\ ${ }^{1}$ Institute of Animal Molecular Biotechnology and Department of Biotechnology, College of Life Sciences and \\ Biotechnology, Korea University, Seoul 02841, Korea \\ ${ }^{2}$ Department of Food and Nutrition, College of Science and Technology, Kookmin University, Seoul 02707, Korea
}

Received December 7, 2020

Revised January 4, 2021

Accepted January 6, 2021

\section{*Correspondence \\ Gwonhwa Song \\ E-mail: ghsong@korea.ac.kr}

ORCID

https://orcid.org/0000-0003-2817-5323

\author{
*Correspondence \\ Whasun Lim \\ E-mail:wlim@kookmin.ac.kr
}

ORCID

https://orcid.org/0000-0002-1328-0465

\begin{abstract}
Dimethachlor is a synthetic herbicide, belonging to the chloroacetanilide group, that inhibits the undesirable growth of weeds via the suppression of very longchain fatty acid synthesis. Although dimethachlor has been shown to run off from agricultural fields into aquatic ecosystems, the toxicity of dimethachlor on aquatic invertebrates and vertebrates is unknown. In our study, we assessed the toxicity of dimethachlor on developing zebrafish embryos by analyzing viability, hatching ability, and phenotypic changes. Embryonic viability decreased from $48 \mathrm{~h}$ post-fertilization (hpf) at the highest concentration of dimethachlor. Decreased hatching ratio, shortened body length, and pathological changes in the eye, heart, and yolk sac were observed at sub-lethal concentrations. Additionally, dimethachlor increased the number of apoptotic cells and level of reactive oxygen species $120 \mathrm{hpf}$. Our results indicate that dimethachlor may act as an anti-developmental toxicant when accumulated in an aquatic environment.
\end{abstract}

Keywords: agriculture, apoptosis, embryonic structures, reactive oxygen species, zebrafish

\section{INTRODUCTION}

Dimethachlor is a chloroacetanilide herbicide that interferes with the synthesis of very long-chain fatty acids (VLCFAs) in target plants (Yang et al., 2010). Because VLCFAs are important lipid components in seeds and surface covering materials, such as plant waxes (von WettsteinKnowles, 1993; Cassagne et al., 1994), compounds of the chloroacetanilide family have been used in crop production for more than 50 years (Junghans et al., 2003). Although these herbicides effectively increase agricultural productivity via the regulation of unwanted weeds, they have caused environmental pollution by leaching. For example, other chloroacetanilides, such as acetochlor, alachlor, and metolachlor, are detectable in surface water samples from rivers and streams (Battaglin et al., 2000). In the case of dimethachlor, its water solubility is $2,300 \mathrm{mg} /$ $\mathrm{L}$; thus, a large amount of dimethachlor dissolves in water (Lewis et al., 2016). As the exposure of aquatic ecosystems to dimethachlor is highly probable because dissolved herbicides can leach with water flow (Ferreira Mendes et al., 2020), it is necessary to identify whether dimethachlor has a harmful effect on aquatic organisms.

To examine the potential risk of a pollutant to aquatic 
An et al. Toxic effects of dimethachlor on embryogenesis of zebrafish

organisms, the zebrafish (Danio rerio) is a widely utilized model organism owing to its high convenience. The high fecundity of zebrafish enables the cheap, rapid, and large-scale toxicity assessment of acute and chronic exposure to chemicals (He et al., 2014). Additionally, this model is used for developmental studies because zebrafish embryos develop outside the maternal body and are transparent (McCollum et al., 2011). For example, zebrafish embryos have been used to test the toxicity of butachlor, which is in the chloroacetanilide family. Butachlor exerts developmental toxicity via interference with the endocrine and immune systems in the embryonic stage (Tu et al., 2013).

In this study, we used zebrafish embryos to determine whether dimethachlor affects embryonic development. We analyzed changes in the viability, hatching, and organogenesis of zebrafish embryos following dimethachlor exposure. Additionally, to elucidate the molecular mechanism of that induces developmental toxicity by dimethachlor, we observed apoptosis and reactive oxygen species (ROS) generation in zebrafish embryos. In summary, our research shows the toxicity of dimethachlor, which increases mortality in zebrafish embryos and suppresses normal organ development at non-lethal concentrations.

\section{MATERIALS AND METHODS}

\section{Acquisition of embryos and chemical treatment}

Wild-type ( $A B$ strain) zebrafish, obtained from the Zebrafish Center for Disease Modeling (KNRRC, Chungnam National University, Korea), were utilized for the acquisition of zebrafish embryos. Parent zebrafish were maintained at $28.5^{\circ} \mathrm{C}$ in a UV-filtered water system with $12 \mathrm{~h}$ light and dark cycles as described in a previous study (Park et al., 2020). To obtain zebrafish eggs, a pair of parent zebrafish were incubated in a spawning box overnight and eggs were laid the next morning after the application of a light stimulus. We washed fertilized eggs twice with an embryo medium and maintained the eggs in a $28^{\circ} \mathrm{C}$ incubator before chemical treatment. For chemical exposure, dimethachlor (Cat No. 45447, Sigma Aldrich, USA) was dissolved in $0.003 \%$ phenylthiourea to avoid pigmentation, which would interfere with the precise detection of the embryo. Embryos exposed to dimethyl sulfoxide (DMSO) were used as a solvent control because the dimethachlor stock was diluted in DMSO.

\section{Evaluation of morphological changes in zebrafish embryos}

Viability, hatching, and morphological changes of embryos in response to dimethachlor exposure were observed under a LEICA DM 2500 microscope (Leica, Germany). Total body length and the relative size of the eye, yolk sac, and pericardial edema were quantified using ImageJ software (NIH, USA).

\section{Detection of apoptotic cells in zebrafish embryos}

The presence of apoptotic cells in zebrafish embryos was investigated after $120 \mathrm{~h}$ of exposure to dimethachlor. We added $5 \mu \mathrm{g} / \mathrm{mL}$ acridine orange (AO; Cat No. A3568, Life Technologies, USA) to dimethachlor-treated media and incubated with embryos at $28^{\circ} \mathrm{C}$ for $1 \mathrm{~h}$. Then, the $\mathrm{AO}$-stained embryos were washed twice and anesthetized. Images of apoptotic cells in embryos were captured under the green fluorescent protein channel of an upright fluorescence microscope (ZEISS, Germany). The number of apoptotic cells was determined using ImageJ software (NIH, USA).

\section{Analysis of ROS production in zebrafish embryos}

The generation of ROS was investigated after $120 \mathrm{~h}$ of exposure to dimethachlor. We added $20 \mu \mathrm{g} / \mathrm{mL}$ dichlorodihydro-fluorescein diacetate (DCFH-DA) to dimethachlor-treated media and incubated with embryos at $28^{\circ} \mathrm{C}$ for $1 \mathrm{~h}$. After incubation, embryos stained with DCFH-DA were washed and anesthetized. ROS production was visualized by green fluorescence, detected using an upright fluorescence microscope (ZEISS, Germany), and measured using ImageJ software (NIH, USA).

\section{Statistical analysis}

Analysis of variance, using the general linear model in the Statistical Analysis System software (SAS Institute, USA), was used to evaluate the significance of data. $p$ values $<0.05$ were considered statistically significant. All experimental data are displayed as means with standard deviations.

\section{RESULTS}

\section{Dimethachlor exerted toxic effects during zebrafish embryo development}

First, we confirmed the viability of zebrafish embryos at 
various concentrations and exposure times of dimethachlor (Fig. 1A). The survival rate of embryos was less than $20 \% 48 \mathrm{~h}$ post-fertilization (hpf) at $100 \mu \mathrm{M}$ and $72 \mathrm{hpf}$ at $75 \mu \mathrm{M}$. Embryos treated with dimethachlor $(50 \mu \mathrm{M})$ showed a $70 \%$ survival rate $120 \mathrm{hpf}$. Furthermore, dimethachlor was toxic even below the lethal concentration. At 48, 72, and $96 \mathrm{hpf}, 50 \mu \mathrm{M}$ dimethachlor-treated eggs showed a significantly lower hatching ratio than vehicletreated eggs ( $p<0.05, p<0.01$; Fig. 1B). Morphological alterations in response to dimethachlor are shown in Fig. 1C. The average body length in the solvent control group
$(3.95 \pm 0.1 \mathrm{~mm})$ was significantly higher than that in the $50 \mu \mathrm{M}$ dimethachlor group $(3.61 \pm 0.17 \mathrm{~mm} ; p<0.001)$, whereas there was no significant difference between the control and $25 \mu \mathrm{M}$ dimethachlor groups (Fig. 1D).

\section{Dimethachlor impaired normal zebrafish embryogenesis}

Next, the effects of dimethachlor on each organ were analyzed by monitoring the morphological alterations of the embryo $120 \mathrm{hpf}$. We analyzed the developmental impairments of dimethachlor on the embryo based on
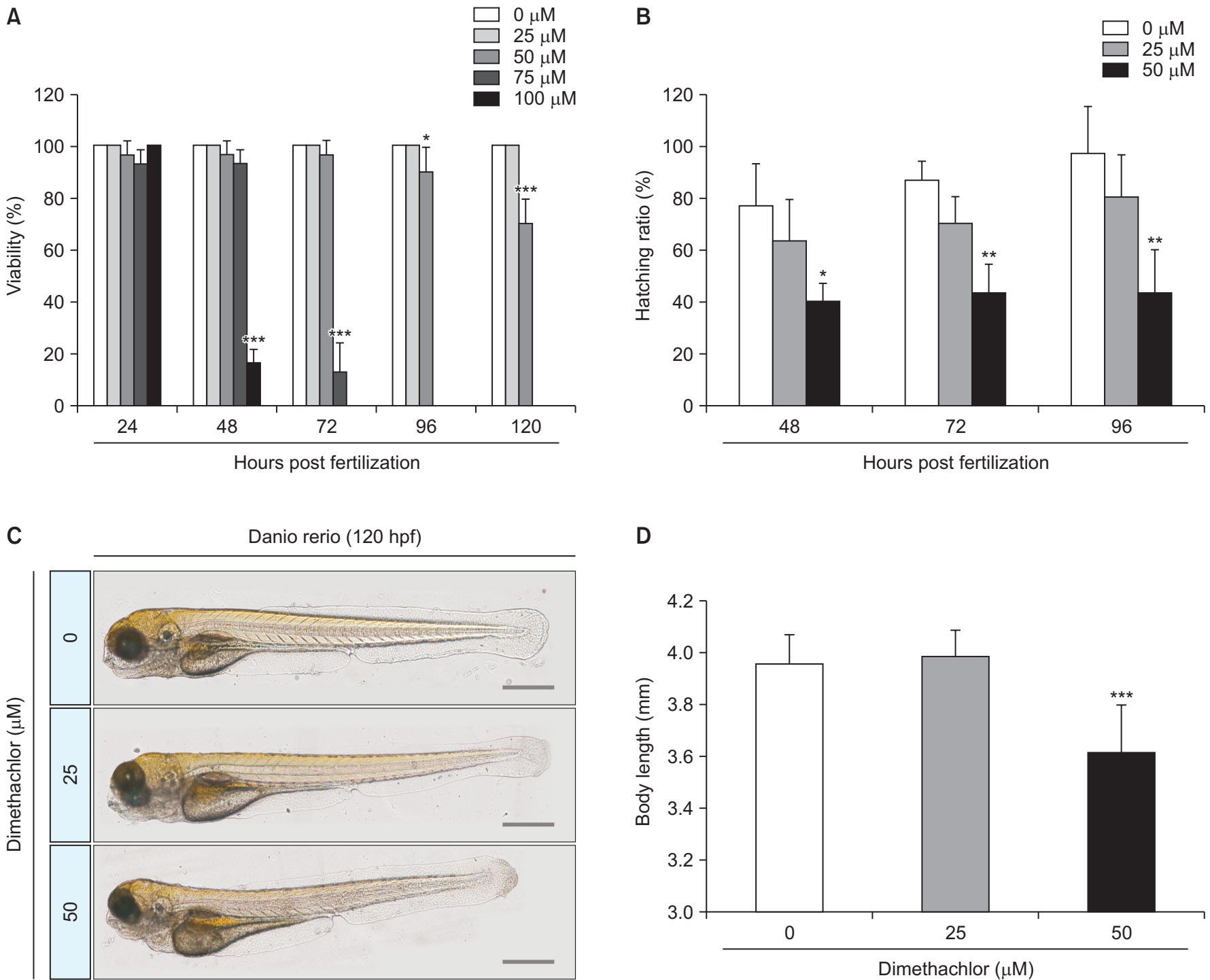

D

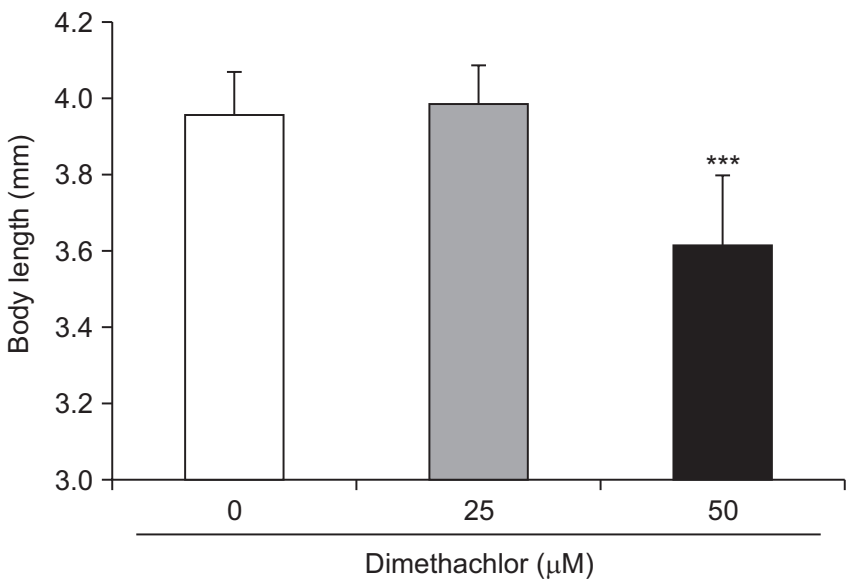

Fig. 1. Adverse effects of dimethachlor on embryo development of zebrafish. (A) Average viability of zebrafish embryos decreased in a dose-dependent and time-dependent manner following dimethachlor exposure. (B) Daily number of hatched eggs between 48 hpf and 96 hpf at each concentration. (C) Representative bright-field images of overall phenotypic change, captured by LEICA DM 2500. Scale bar: $500 \mu \mathrm{m}$. (D) Total body length was quantified using "Length" in ImageJ software, $p$-values that indicate significant differences: ${ }^{*} p<0.05$, ${ }^{* *} p<0.01$, and ${ }^{* * *} p<0.001$. 
A

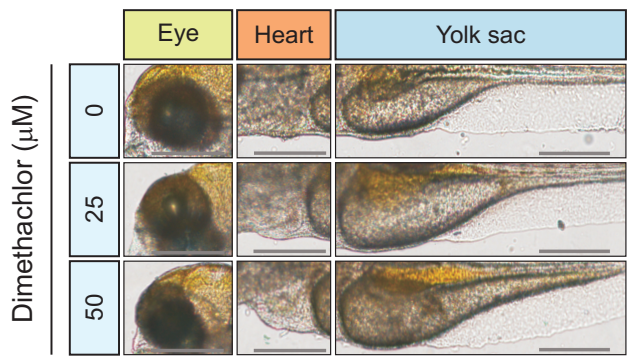

B

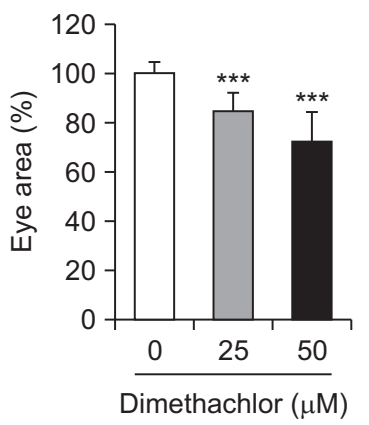

C

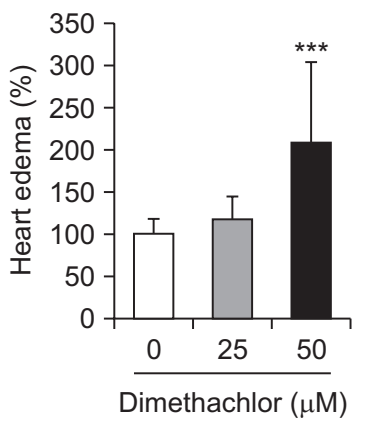

D

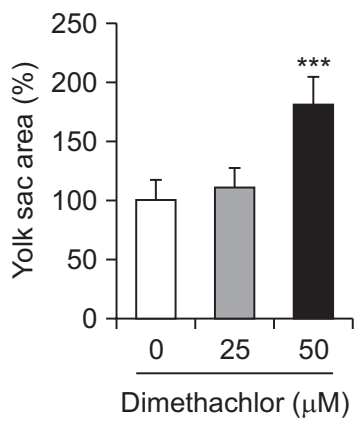

Fig. 2. Phenotypic change of embryos exposed to dimethachlor $120 \mathrm{hpf}$. (A) Magnified images of specific regions show abnormalities in response to different concentrations of dimethachlor. Area of eye (B), pericardiac edema (C), and yolk sac (D) was measured using "Area" in ImageJ software and is displayed as a percentage relative to the solvent group. Scale bar: $500 \mu \mathrm{m}, p$-value that indicates significant difference: ${ }^{* * *} p<0.001$.

A

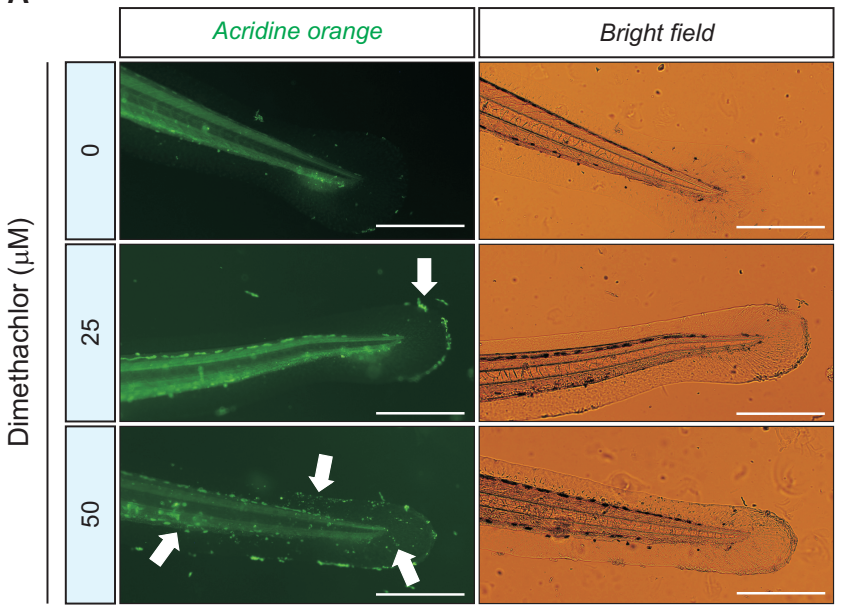

B

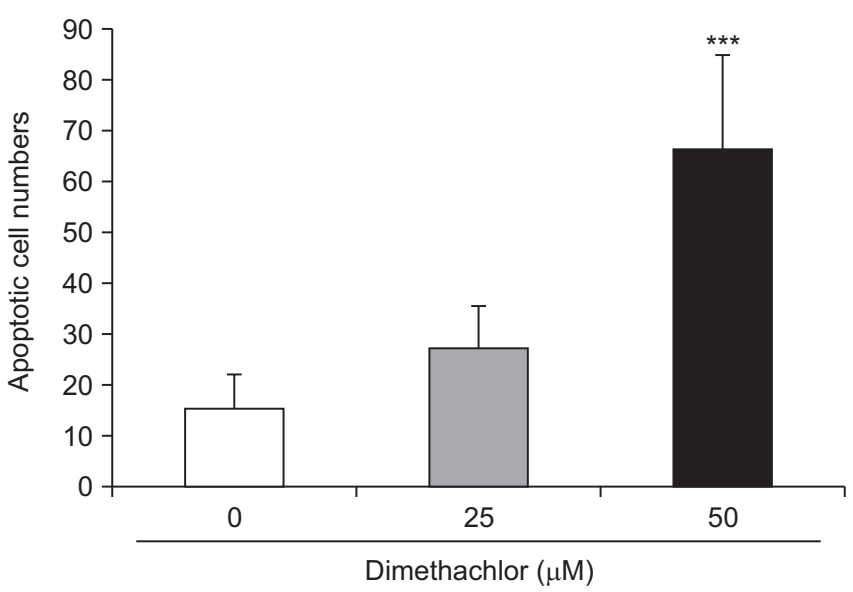

Fig. 3. Detection of apoptotic cells in dimethachlor-exposed embryos. (A) Apoptosis was highly elevated in the posterior region. Each green dot, indicated by white arrows, represents an apoptotic cell. Scale bar: $500 \mu \mathrm{m}$. (B) Number of apoptotic cells was measured using "Find Maxima" in ImageJ software, $p$-value that indicates significant difference: ${ }^{* * *} p<0.001$.

changes to the eye, heart, and yolk sac (Fig. 2A). Eye deformation was observed in the $25 \mu \mathrm{M}$ dimethachlor group $(p<0.001)$; the size of the eye was $84 \%$ and $72 \%$ lesser in the $25 \mu \mathrm{M}$ and $50 \mu \mathrm{M}$ dimethachlor groups, respectively, than that in the solvent control group (Fig. 2B). Moreover, the cardiotoxicity of dimethachlor was confirmed by measuring the size of the pericardial edema (Fig. 2C). When exposed to $50 \mu \mathrm{M}$ dimethachlor, the pericardial edema was twice the size of that in the solvent control group $(p<0.001)$. Furthermore, the relative area of the yolk sac was approximately $180 \%$ higher in the $50 \mu \mathrm{M}$ dimethachlor group than that in the solvent control group $(p<0.001$, Fig. 2D). Taken together, these results indicate that dimethachlor disrupts normal organ development in the early stage of zebrafish development.

\section{Dimethachlor induced apoptosis and ROS in zebrafish embryos}

To clarify the underlying mechanisms of developmental suppression by dimethachlor, zebrafish embryos were stained with AO fluorescent dye. The number of apoptotic cells, visualized with green dots, was highest in the posterior region of dimethachlor-exposed embryos (Fig. $3 \mathrm{~A}$ ), not markedly highlighted in the anterior body (data not shown). The number of apoptotic cells in the solvent control group (15.25 \pm 6.13) was more than 1.5- and 4.0fold lower than that in the $25 \mu \mathrm{M}$ and $50 \mu \mathrm{M}$ dimethachlor groups, respectively (Fig. 3B). Furthermore, we observed organ malformation in eye, heart and yolk sac in the previous results. Because induction of oxidative stress is a major cause of organ malformation, we monitored 
ROS levels in this region. The levels of ROS increased in a concentration-dependent manner in the anterior region of dimethachlor-exposed embryos (Fig. 4A). The level of ROS, indicated by the relative green intensity of DCFHDA-stained cells, was $164 \pm 32 \%$ higher in the $25 \mu \mathrm{M}$ dimethachlor group and $252 \pm 68 \%$ higher in the $50 \mu \mathrm{M}$ dimethachlor group than that in the solvent control group (Fig. 4B). These experiments indicate that the embryotoxicity of dimethachlor is mediated by apoptosis and oxidative stress.

\section{DISCUSSION}

Dimethachlor, a chloroacetanilide, selectively exerts herbicidal activity on pre-emergent weeds via the inhibition of FA synthesis (Yang et al., 2010). Because of its high-water solubility, dimethachlor has the potential to unintentionally contaminate the water environment. However, few studies have investigated the non-target toxicity of dimethachlor, especially on aquatic organisms. For this reason, we evaluated the toxicity of dimethachlor by analyzing the viability, hatching ability, and morphological alteration of zebrafish embryos. We showed that dimethachlor disrupts normal developmental processes via the induction of apoptosis and ROS production.

First, dimethachlor showed acute lethal toxicity. At a concentration of $100 \mu \mathrm{M}$, the death of zebrafish embryos was observed from $48 \mathrm{hpf}$ and 100\% of embryos died 72 hpf. Furthermore, dimethachlor also reduced hatching ability. Normally, zebrafish embryos hatch from the chorion within 48-72 hpf by enzymatic processes and spontaneous movements (Kimmel et al., 1995; Capriello et al., 2019). Therefore, decrease in hatching ratio and delayed time of hatching is one of the criteria to estimate the developmental toxicity (Chakraborty et al., 2016; Chahardehi et al., 2020). Zebrafish eggs exposed to $25 \mu \mathrm{M}$ and 50 $\mu \mathrm{M}$ dimethachlor showed decrease in hatching ability, suggests that dimethachlor also negatively affected embryogenesis at sub-lethal concentrations.

Additionally, dimethachlor-exposed embryos displayed abnormal phenotypic transformation, such as a shortened body length, decreased eye size, pericardial edema, and increased yolk sac size, that are common characteristics in response to toxic substances. First, decreased eye size is a representative phenotypic change related to extensive eye malformations such as growth retardation of eye and irregular cell death in retinal region during ocular development (Malicki et al., 1996). For example, deformation of retinal structure induced by apoptosis in phenanthrene exposed embryos accompanied with alteration of eye diameter (Huang et al., 2013). Pericardiac edema is a representative indicator of cardiotoxicity, usually accompanied by an irregular heart rate and deformation of the heart and circulatory system (Liang et al., 2016; Zakaria et al., 2018). The yolk sac is the storehouse of proteins and lipids and provides nutrition in the early stage of zebrafish
A

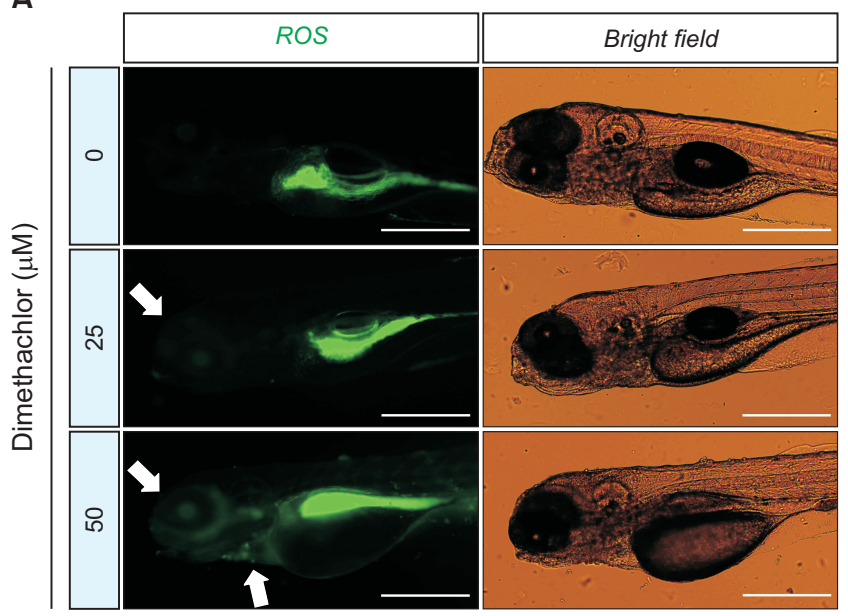

B

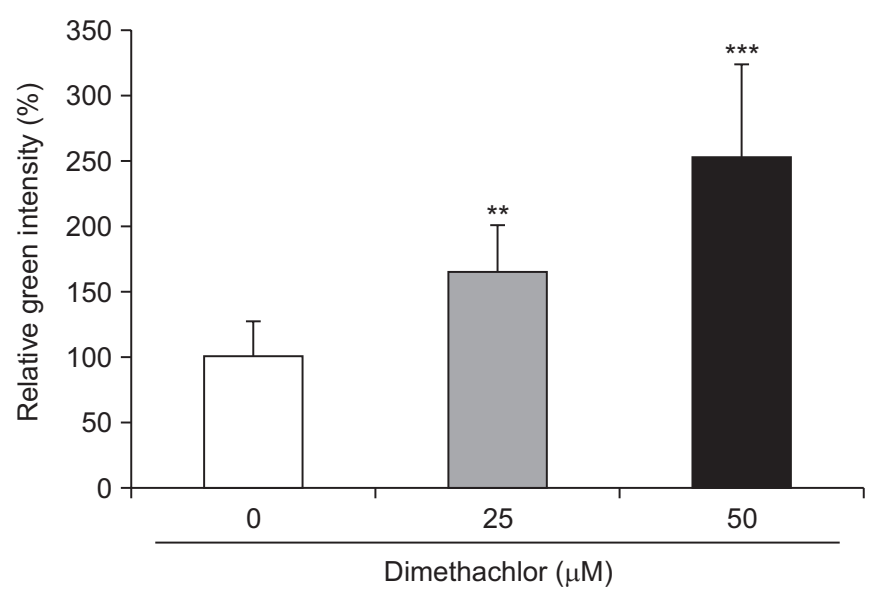

Fig. 4. Detection of ROS in dimethachlor-exposed embryos. (A) ROS levels increased in the anterior region. White arrows indicate regions where ROS levels markedly increased. Scale bar: $500 \mu \mathrm{m}$. (B) Green fluorescence intensity of DCFH-DA was quantified using "RawlntDen" in ImageJ software and is displayed as a percentage relative to the solvent group. $p$-values that indicate significant differences: ${ }^{* *} p<0.01$ and ${ }^{* * *} p<0.001$. DCFH-DA, dichloro-dihydro-fluorescein diacetate; ROS, reactive oxygen species. 
An et al. Toxic effects of dimethachlor on embryogenesis of zebrafish

embryo development before the uptake of external feed. Therefore, an enlarged yolk sac generally indicates that the yolk sac is not functioning properly (Sant and TimmeLaragy, 2018). These results suggest that impaired embryogenesis, induced by dimethachlor at an early stage of embryogenesis, prevents the development of embryos into normal juveniles and adults.

Next, we detected apoptotic cells in the posterior body of dimethachlor-exposed embryos using AO staining. During embryonic development, programmed cell death at a suitable time and site is essential for organ formation. For example, the rate of apoptosis changes at various times in each part of the brain and apoptotic cells are mainly detected between $20 \mathrm{hpf}$ and $48 \mathrm{hpf}$ in the lens and $18 \mathrm{hpf}$ and $24 \mathrm{hpf}$ in tailbuds of zebrafish (Cole and Ross, 2001). Thus, the increased number of apoptotic cells in the tail $120 \mathrm{hpf}$ represents irregular cell death. This result is in conjunction with body length shortening by dimethachlor. Also, elevated apoptosis might result in defects in the vascular and neural systems in the posterior regions, which negatively affects whole embryogenesis (Eum et al., 2016; Chen et al., 2017). Dimethachlor generated not only apoptotic cells but also ROS, which adversely affected embryonic organogenesis. For instance, the generation of ROS caused by trimethyltin chloride obstructs the normal function of the zebrafish eye via apoptosis in the retinal region (Kim et al., 2019). Additionally, there are previous studies on several proteins that mediate oxidative stress-induced cardiac dysfunction, such as the aromatic hydrocarbon receptor and HECT domain and ankyrin repeat-containing E3 ubiquitin-protein ligase 1 (Razaghi et al., 2018; Ren et al., 2020). Therefore, ROS generation in the anterior region of the embryo by dimethachlor might be the cause of the abnormal organogenesis identified in the previous results.

\section{CONCLUSION}

To investigate the toxic effects of dimethachlor, we utilized zebrafish embryos for a developmental toxicity assessment. Dimethachlor reduced viability in the early stage of development and induced defects in the eye, heart, and yolk sac in the embryo. These effects were caused by apoptosis and oxidative stress following dimethachlor exposure. In conclusion, to our knowledge, this is the first study to reveal the developmental toxicity of dimethachlor and its mechanisms in zebrafish embryos.

\section{CONFLICTS OF INTEREST}

No potential conflict of interest relevant to this article was reported.

\section{ACKNOWLEDGEMENTS}

This research was supported by a grant of the National Research Foundation of Korea (NRF) grant funded by the Ministry of Science and ICT (MSIT) [grant number 2018R1C1B6009048].

\section{AUTHOR CONTRIBUTIONS}

\author{
Conceptualization: G.S. and W.L. \\ Methodology: G.A., and H.P. \\ Investigation: G.A., and H.P. \\ Data curation: G.S. and W.L. \\ Visualization: G.A. \\ Funding: G.S., and W.L. \\ Writing-original draft preparation: G.A. \\ Writing-review and editing: G.S. and W.L. \\ Supervision: G.S. and W.L.
}

\section{AUTHOR'S POSITION AND ORCID NO.}

G An, Graduate Student, https://orcid.org/0000-0002-0065-793X

H Park, Graduate Student, https://orcid.org/0000-0001-9876-070X

G Song, Professor, https://orcid.org/0000-0003-2817-5323

W Lim, Assistant Professor, https://orcid.org/0000-0002-1328-0465

\section{REFERENCES}

Battaglin WA, Furlong ET, Burkhardt MR, Peter CJ. 2000. Occurrence of sulfonylurea, sulfonamide, imidazolinone, and other herbicides in rivers, reservoirs and ground water in the Midwestern United States, 1998. Sci. Total Environ. 248:123133.

Capriello T, Grimaldi MC, Cofone R, D'Aniello S, Ferrandino I. 2019. Effects of aluminium and cadmium on hatching and swimming ability in developing zebrafish. Chemosphere 
222:243-249.

Cassagne C, Lessire R, Bessoule JJ, Moreau P, Creach A, Schneider F, Sturbois B. 1994. Biosynthesis of very long chain fatty acids in higher plants. Prog. Lipid Res. 33:55-69.

Chahardehi AM, Arsad H, Lim V. 2020. Zebrafish as a successful animal model for screening toxicity of medicinal plants. Plants (Basel) 9:1345.

Chakraborty C, Sharma AR, Sharma G, Lee SS. 2016. Zebrafish: a complete animal model to enumerate the nanoparticle toxicity. J. Nanobiotechnology 14:65.

Chen X, Dong Q, Chen Y, Zhang Z, Huang C, Zhu Y, Zhang Y. 2017. Effects of Dechlorane Plus exposure on axonal growth, musculature and motor behavior in embryo-larval zebrafish. Environ. Pollut. 224:7-15.

Cole LK and Ross LS. 2001. Apoptosis in the developing zebrafish embryo. Dev. Biol. 240:123-142.

Eum J, Kwak J, Kim HJ, Ki S, Lee K, Raslan AA, Park OK, Chowdhury MA, Her S, Kee Y, Kwon SH, Hwang BJ. 2016. 3D visualization of developmental toxicity of 2,4,6-trinitrotoluene in zebrafish embryogenesis using light-sheet microscopy. Int. J. Mol. Sci. 17:1925.

He JH, Gao JM, Huang CJ, Li CQ. 2014. Zebrafish models for assessing developmental and reproductive toxicity. Neurotoxicol. Teratol. 42:35-42.

Huang L, Wang C, Zhang Y, Wu M, Zuo Z. 2013. Phenanthrene causes ocular developmental toxicity in zebrafish embryos and the possible mechanisms involved. J. Hazard. Mater. 261:172-180.

Junghans M, Backhaus T, Faust M, Scholze M, Grimme LH. 2003. Predictability of combined effects of eight chloroacetanilide herbicides on algal reproduction. Pest Manag. Sci. 59:1101-1110.

Kim J, Kim CY, Oh H, Ryu B, Kim U, Lee JM, Jung CR, Park JH. 2019. Trimethyltin chloride induces reactive oxygen speciesmediated apoptosis in retinal cells during zebrafish eye development. Sci. Total Environ. 653:36-44.

Kimmel CB, Ballard WW, Kimmel SR, Ullmann B, Schilling TF. 1995. tages of embryonic development of the zebrafish. Dev. Dyn. 203:253-310.

Lewis KA, Tzilivakis J, Warner DJ, Green A. 2016. An international database for pesticide risk assessments and manage- ment. Hum. Ecol. Risk Assess. 22:1050-1064.

Liang J, Jin W, Li H, Liu H, Huang Y, Shan X, Li C, Shan L, Efferth T. 2016. In vivo cardiotoxicity induced by sodium aescinate in zebrafish larvae. Molecules 21:190.

Malicki J, Neuhauss SC, Schier AF, Solnica-Krezel L, Stemple DL, Stainier DY, Abdelilah S, Zwartkruis F, Rangini Z, Driever W. 1996. Mutations affecting development of the zebrafish retina. Development 123:263-273.

McCollum CW, Ducharme NA, Bondesson M, Gustafsson JA. 2011. Developmental toxicity screening in zebrafish. Birth Defects Res. C Embryo Today 93:67-114.

Park S, Song G, Lim W. 2020. Anti-developmental effects of imazosulfuron on zebrafish embryos during development. J. Anim. Reprod. Biotechnol. 35:28-34.

Razaghi B, Steele SL, Prykhozhij SV, Stoyek MR, Hill JA, Cooper MD, McDonald L, Lin W, Daugaard M, Crapoulet N, Chacko S, Lewis SM, Scott IC, Sorensen PHB, Berman JN. 2018. hacel Influences zebrafish cardiac development via ROSdependent mechanisms. Dev. Dyn. 247:289-303.

Ren F, Ji C, Huang Y, Aniagu S, Jiang Y, Chen T. 2020. AHRmediated ROS production contributes to the cardiac developmental toxicity of PM2.5 in zebrafish embryos. Sci. Total Environ. 719:135097.

Sant KE and Timme-Laragy AR. 2018. Zebrafish as a model for toxicological perturbation of yolk and nutrition in the early embryo. Curr. Environ. Health Rep. 5:125-133.

Tu W, Niu L, Liu W, Xu C. 2013. Embryonic exposure to butachlor in zebrafish (Danio rerio): endocrine disruption, developmental toxicity and immunotoxicity. Ecotoxicol. Environ. Saf. 89:189-195.

von Wettstein-Knowles PM. 1993. Waxes, cutin and suberin. In: Moore TS (Ed.), Lipid Metabolism in Plants. CRC Press, Boca Raton, pp. 127-166.

Yang X, Guschina IA, Hurst S, Wood S, Langford M, Hawkes T, Harwood JL. 2010. The action of herbicides on fatty acid biosynthesis and elongation in barley and cucumber. Pest Manag. Sci. 66:794-800.

Zakaria ZZ, Benslimane FM, Nasrallah GK, Shurbaji S, Younes NN, Mraiche F, Da'as SI, Yalcin HC. 2018. Using zebrafish for investigating the molecular mechanisms of drug-induced cardiotoxicity. Biomed Res. Int. 2018:1642684. 